\title{
The levator ani muscle repair: a call to action
}

\author{
R. Tomashev ${ }^{1,2,3} \cdot$ S. Abbas Shobeiri ${ }^{1,2,3}$
}

Published online: 23 June 2021

(c) Springer Nature Switzerland AG 2021

The ailments of the levator ani muscle (LAM) have entertained surgeons for generations. The 1808 book on the principles of midwifery by John Burns recognized extreme levator ani injuries associated with symphysis separation. About 1878 , Schatz was one of the first to call attention to repair of lacerations to the levator ani muscles, and the ill effects which followed the loss of muscular support. In 1899, ML Harris lamented a lack of knowledge of the anatomy of the female pelvis in everyday use textbooks described consequences of LAM loss as it pertains to pelvic floor disorders. In 1903, McGavin described a perineal hernia as a result of the LAM defect in a multiparous woman. He pondered about the etiology of the problem and tried to diagnose the problem clinically, very much similar to the clinical evaluation done today. Reading his accounts, if we had performed an endovaginal ultrasound today, it is likely that we would have found an iliococcygeal tear with herniation of bowel into the ischiorectal fossa. Dr. McGavin intended to endeavor to cure the hernia by the implantation of a "spider's web" filigree of silver wire in the floor of the pelvis." In 1908, Fothergill, in the proceeding of the royal society of medicine stated, "It was useless to try and restore the action of a pelvic floor in which the muscle was already cicatricial and permanently damaged; it was not possible to dissect out and restore by surgical measures a muscle which was, even in the body of a healthy normal female, difficult to expose and manipulate. If the muscle were damaged, then it seemed to him the surgeon must fall back on the fascial and non-striated muscle supports, and these must be shortened and strengthened." The surgical approach of reconstituting fascia and leaving

\footnotetext{
S. Abbas Shobeiri

Abbas.Shobeiri@inova.org

http://www.urogynecologist.com

Virginia Commonwealth University, Richmond, USA

2 George Mason University, Fairfax, USA

3 Department of Obstetrics and Gynecology, INOVA Women's Hospital, 3300 Gallows Rd, 2nd floor, Falls Church, VA 22042, USA
}

the torn muscles alone has continued to this day, and examples are vaginal tapes and any colpopexy surgery aiming at reinforcing lax connective tissue. The exception is the Harris procedure described in the late 1800s, which described the repair of the levator ani muscles: "The procedure was described which is the shortening of the torn or stretched fibers of the levator ani muscles, with a slight modification... dissect out a triangular flap along each lateral sulcus. The base of each triangular denudation is toward the vaginal orifice.... Now dissect out the levator ani muscle at the side of the rectum, seize its anterior fibers and draw on them to see how much slack needs to be taken up in order to hold the rectum and perineal body forward under the pubis. When sufficient of this muscle is free, fold it upon itself in its longitudinal course and stitched the redundant tissue together with interrupted or continuous catgut suture, thus trying the rectum and perineum well up (Fig. 1)" [1].

The Harris procedure was buried in the annals of history until Ris et al. [2] reported their experience in the current series. Ris et al.'s case series performed over 18 years cannot be judged based on pelvic floor ultrasonography which has become standard recently for the diagnosis of specific LAM subdivision injuries [3]. What is striking and what Ris et al. should be congratulated on is that they have used curiosity, clinical acumen, and patient symptoms to seek innovative solutions for very prevalent yet untreated problem of the LAM injury. There are problems in this study inherent to the gradual experience of the authors with the technique, which shall not be emphasized.

When we have presented our experience with various approaches to LAM repair, the audience has frequently asked how the repair of these muscles, even if possible, would benefit the patient. Reconstituting the LAM subdivisions may be necessary for recreation and proper function of the levator plate. Patients with LAM disruption, elongation, or deficiency lack normal anorectal plate function and present with anorectal dysfunction [4]. We strongly recommend endovaginal 3D pelvic floor ultrasonography as there is an odds ratio of 8.3 for pelvic floor disorders development when both levator defect and tissue failures are 


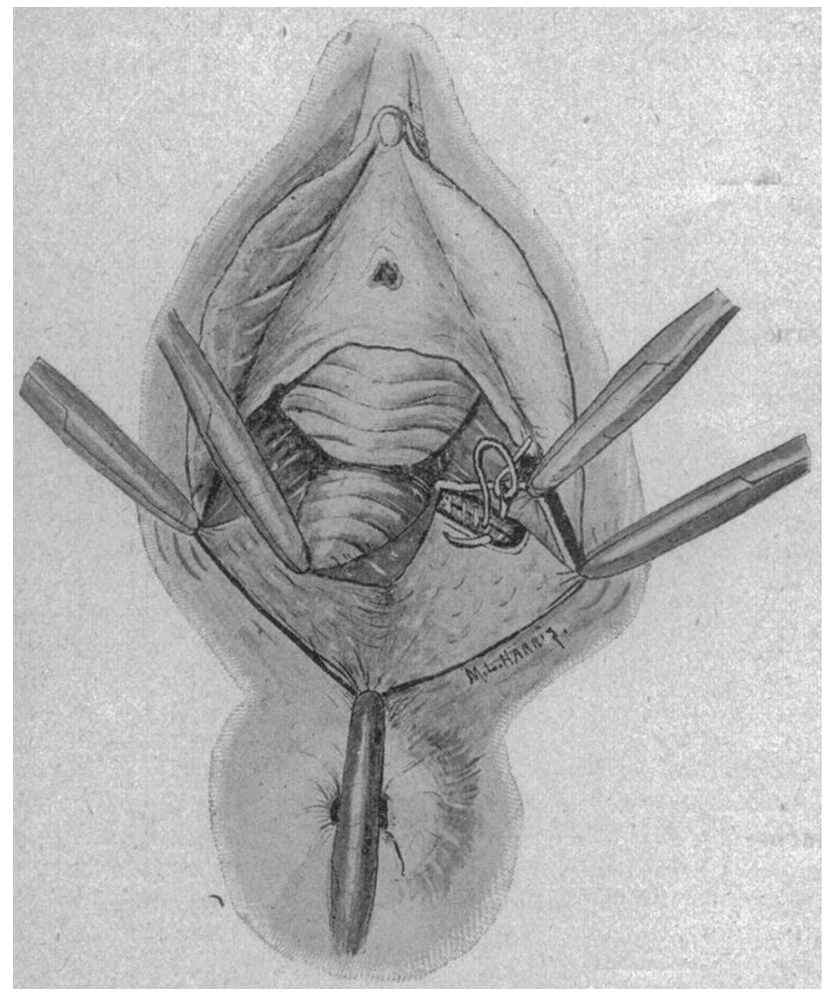

Fig. 1 From Harris: muscle drawn together with buried catgut suture (the repair of old lacerations of the pelvic floor, 1899)

present [5]. LAM defects appear to be a necessary condition for architectural distortions of pelvic floor to occur [6] and $3 \mathrm{D}$ endovaginal ultrasound is the most sensitive and readily available modality for visualization of the LAM subdivisions $[3,7]$. On the other hand, a recent long-term study of patients 6-17 years from delivery utilizing perineal pelvic floor ultrasound revealed that although LAM avulsion was strongly associated with anterior compartment defects, the relationship between avulsion and other pelvic floor disorders was not significant [8]. If we believe that the LAM defects are asymptomatic after vaginal delivery and their long-term association with pelvic floor disorders are questionable, should the defects still be repaired? I personally believe that it is a disservice to the patient to wait until they develop prolapse, pain, voiding, or defecatory dysfunction before the levator muscles are repaired. Although it is intuitive that a disrupted LAM should be reattached for proper pelvic floor function, any technique developed to repair the levator ani muscle injuries should be standardized and followed long-term with both endovaginal ultrasound and validated patient reported outcomes to assess objective and subjective changes.

Research has been slow in the area of postpartum LAM injury detection, because: (1) all postpartum patients are understandably sore in the vaginal area and LAM trauma symptoms are at times the same as a normal vaginal delivery,
(2) immediately after delivery, about $40 \%$ of patients have levator ani hematomas indicative of injury and in 6 months after delivery $13 \%$ of these defects persist [9-11]. Currently, delineating which patients have the genetic capacity to heal their LAM muscles is lacking. Research and progress in LAM repair has been hindered, because it requires mastery of: (1) the pelvic floor anatomy, (2) the surgical acumen, (3) clinical insight, and (4) the core competency in point-of-care imaging to detect LAM injuries. Our graduating fellows and practicing physicians need to master these four elements. Almost 150 years have passed since the first known report of the repair of the LAM. Learning how to precisely repair LAM injuries is the key to total correction of the pelvic floor anatomical distortions to replace the current pelvic floor surgeries that currently aim to correct the facial defects without even attempting to determine the sub-vaginal intricate LAM status.

\section{Declarations}

Conflict of interest The authors declare that they have no conflict of interest.

Ethical approval This article does not contain animal or human subjects involved in any part of the study and therefore does not require formal ethical approval.

Informed consent For this kind of study, formal informed consent is not required.

\section{References}

1. Newman HP (1900) Improved technique in major and minor surgery of the female generative organs. JAMA 35:268-269

2. Ris F, Alketbi M, Scarpa CR et al (2019) Levator ani repair by transvaginal approach. Tech Coloproctol 23:167-169. https://doi. org/10.1007/s10151-018-1907-x

3. Shobeiri SA, Leclaire E, Nihira MA, Quiroz LH, O'Donoghue D (2009) Appearance of the levator ani muscle subdivisions in endovaginal three-dimensional ultrasonography. Obstet Gynecol 114:66-72

4. Rostaminia G, Javadian P, Awad C, Shobeiri SA (2019) Ultrasound indicators of rectal support defect in women with obstructive defecatory symptoms. Female Pelvic Med Reconstr Surg 25(3):222-225. https://doi.org/10.1097/SPV.0000000000000511

5. DeLancey JOL, Morgan DM, Fenner DE, Kearney R, Guire K, Miller JM et al (2007) Comparison of levator ani muscle defects and function in women with and without pelvic organ prolapse. Obstet Gynecol 109(2 Pt 1):295-302

6. Huebner M, Margulies RU, DeLancey JOL (2008) Pelvic architectural distortion is associated with pelvic organ prolapse. Int Urogynecol J 19(6):863-867

7. Rostaminia G, White D, Hegde A, Quiroz LH, Davila GW, Shobeiri SA (2013) Levator ani deficiency and pelvic organ prolapse severity. Obstet Gynecol 121:1017-1024 
8. Rostaminia G, Peck JD, Quiroz LH, Shobeiri SA (2015) How well can levator ani muscle morphology on 3D pelvic floor ultrasound predict the levator ani muscle function? Int Urogynecol J 26(2):257-262

9. Handa VL, Blomquist JL, Roem J, Muñoz A, Dietz HP (2019) Pelvic floor disorders after obstetric avulsion of the levator ani muscle. Female Pelvic Med Reconstr Surg 25(1):3-7. https://doi. org/10.1097/SPV.0000000000000644

10. Van Delft K, Shobeiri SA, Sultan AH, Thakar R (2012) Haematomas may masquerade as levator ani muscle defects. Int Urogynecol J 23(2):S43-S244
11. van Delft K, Thakar R, Shobeiri SA, Sultan AH (2014) Levator haematoma at the attachment zone as an early marker for levator ani muscle avulsion. Ultrasound Obstet Gynecol 43(2):210-217

Publisher's Note Springer Nature remains neutral with regard to jurisdictional claims in published maps and institutional affiliations. 